\title{
Axiomatic and Dynamic Processes for a Symmetric Allocation Rule
}

\author{
Chia-Hung Li ${ }^{1}$, Jo-Wei Chiang ${ }^{2, *}$, En-Cheng Chi ${ }^{1, *}$ and Yu-Hsien Liao ${ }^{3, *}$ \\ 1 Office of Physical Activities, National Pingtung University, Pingtung County 90003, Taiwan; \\ pippen0917@mail.nptu.edu.tw \\ 2 Department of Adult and Continuing Education, National Chung Cheng University, Chiayi 621301, Taiwan \\ 3 Department of Applied Mathematics, National Pingtung University, Pingtung County 900391, Taiwan \\ * Correspondence: vicky5433@alum.ccu.edu.tw (J.-W.C.); g731007@mail.nptu.edu.tw (E.-C.C.); \\ twincos@mail.nptu.edu.tw (Y.-H.L.)
}

Citation: Li, C.-H.; Chiang, J.-W.;

Chi, E.-C.; Liao, Y.-H. Axiomatic and

Dynamic Processes for a Symmetric Allocation Rule. Symmetry 2021, 13, 1474. https://doi.org/10.3390/ sym13081474

Academic Editor: Juan Luis García Guirao

Received: 14 July 2021

Accepted: 9 August 2021

Published: 11 August 2021

Publisher's Note: MDPI stays neutral with regard to jurisdictional claims in published maps and institutional affiliations.

Copyright: (c) 2021 by the authors. Licensee MDPI, Basel, Switzerland. This article is an open access article distributed under the terms and conditions of the Creative Commons Attribution (CC BY) license (https:/ / creativecommons.org/licenses/by/ $4.0 /)$.
Abstract: It has recently become imperative to analyze relevant issues to improve the efficiency of resource allocation by means of different perspectives and ways of thinking. There exist numerous decisive factors, such as changes in forms of allocation, reactive behavior, and the interaction and functional effectiveness of strategies, that need to be complied. In contrast to expert meetings, rules of thumb, or other existing concepts, this article aims to offer a different and efficient resource allocation approach by applying game-theoretical methods to resource-allocation situations. Our major investigative procedures are as follows: (1) after comparing our method with pre-existing allocation rules from pre-existing allocation rules, a symmetric allocation rule is proposed that considers both units and their energy grades; (2) based on the properties of grade completeness, criterion for models, unmixed equality symmetry, grade synchronization, and consistency, some axiomatic outcomes are used to examine the mathematical accuracy and the applied rationality of this symmetric allocation rule; (3) based on a symmetrical revising function, a dynamic process is applied to show that this symmetric allocation rule can be reached by units that start from an arbitrary grade completeness situation; and (4) these axiomatic and dynamic results and related meanings are applied to show that this symmetric allocation rule can present an optimal alternative guide for resource-allocation processes. Related applications, comparisons, and statements are also offered throughout this article.

Keywords: unit; energy grade; symmetric allocation rule; axiomatic and dynamic result

\section{Introduction}

For a long time, it has been an important aim for many investigators to research how to efficiently operate resource-allocation processes. An apposite resource-allocation concept that allows the proper distribution of limited resources to the most needed links and for the most efficient aims to be achieved is required. On the other hand, game-theoretical results can be adopted to analyze many processes with interactive phenomena by applying various mathematical fields, leading to apposite results that are simultaneously acceptable, correct, feasible and rational. This approach further includes the analysis and establishment of how to apply the allocation mechanism to interactive processes, such as the operation of resource allocation, the proportional distribution of strategy implementation, and so on. Therefore, game-theoretical results are widely applied in various fields, such as biochemical engineering, economic analysis, environmental sustainability, management science, policy formulation, and so on. For instance, the core is the set of related outcomes that exhibit efficient and coalitional rationality in resource-allocation processes. Shapley [1] defined the Shapley value to analyze resource-allocation processes by collecting the entire expected operational value for each unit. Ransmeier [2] proposed the equal allocation non-separable cost (EANSC) to evaluate the optimum yield for dams managed by the Tennessee Valley 
Authority. Under the distributing notion of the EANSC, units first receive their marginal effects and then distribute the remaining resources equally. Later, Hsieh and Liao [3] first used the individual rule to define the PEANSC and then applied a reduced model and its consistency to demonstrate that the pseudo equal allocation non-separable cost (PEANSC) is a fair allocation rule that satisfies several useful axioms. Under the distribution concept of the PEANSC, units first receive their individual effects and further distribute the remaining resources equally. The primary discrimination is that the EANSC arises from the "marginal effects" of units, and the PEANSC originates from the "individual effects" of units.

Under traditional side-payment models, a mensuration map is defined by considering all the groups formed by participating units. This means that the behaviors available for each unit are either to act fully in a situation or not to act at all. In the real world, however, allocation often varies relative to other parties in response to the rapidly changing interactions among units, groups, and conditions. Each unit will be provided with a certain quantity of energy grades, and thus its ability might be different from other units. Therefore, a multi-choice side-payment model could be regarded as an extension of a traditional model in which each unit presents various energy grades. Several allocation rules have been discussed for multi-choice models. By calculating the whole effects for a given unit under multi-choice clan models, Branzei [4] proposed some extended core concepts by adopting domination among units and its energy grades. Liao [5] introduced an extended EANSC by applying the maximal pure effects of all units among their energy grades; Nouweland et al. [6] defined an analogue of the Shapley value [1] by considering the replicated behavior among units among their energy grades. On the other hand, Hwang and $\mathrm{Li}$ [7] defined an extended core by simultaneously focusing on the units and their energy grades under multi-choice consideration.

On the strength of the above discussion, the motivation for this article is as follows: as mentioned above, the core, the Shapley value, and the EANSC have been extended to the framework of multi-choice models. We investigate whether the PEANSC could also be extended to become the most efficient resource-allocating mechanism under multichoice consideration.

This article is devoted to answering this question. The main outcomes are as follows.

- Inspired by Hsieh and Liao [3] and Hwang and Li [7], the cumulative individual rule (CIDR) is considered for multi-choice side-payment models by simultaneously focusing on the units and their energy grades in Section 2.

- With the aim of determining the mathematical accuracy and the applied rationality of the CIDR, two axiomatic outcomes are used to show that the CIDR is the only allocation rule that satisfies grade completeness, criterion for models, unmixed equality symmetry, grade synchronization, and consistency in Section 3.

- In real-world situations, however, many allocation outcomes show less than expected outcomes in the initial stage of allocating processes. Therefore, this article attempts to adopt dynamic analysis to establish an interactive process that can be revised at any time for resource-allocation processes. In Section 4, a symmetric revision function and relevant dynamic process are defined to show that the CIDR can be reached by units who start from an arbitrary grade completeness outcome.

- In conjunction with axiomatic and dynamic processes related to resource-allocation processes in real-world situations, the applicability and the plausibility of the CIDR are further analyzed in Section 5. Related applications and statements are also offered throughout this article.

\section{Preliminaries}

To define a new allocation rule in the framework of multi-choice models, some notations and definitions are necessary to present in this section. Further, a motivating example and a real illustration example are also provided.

Let $E U$ be the universe of units; for example, the set formed by people on the Earth. Any $x \in E U$ is said to be a unit of $E U$; for example, a person on the Earth. For $x \in E U$ 
and $g_{x} \in \mathbb{N}, G_{x}=\left\{0,1, \cdots, g_{x}\right\}$ could be treated as the energy grade space of unit $x$ and $G_{x}^{+}=G_{x} \backslash\{0\}$, where 0 denotes no participation. Assume that $U \subseteq E U$ is the largest set of all units of an interactive process in $E U$; for example, all people in a country. Let $G^{U}=\prod_{x \in U} G_{x}$ be the product set of the energy grade (action) spaces of all units of $U$. Denote $0_{U}$ as the zero vector in $\mathbb{R}^{U}$.

A multi-choice model is a triple $(U, g, E)$, where $U \neq \varnothing$ is a finite set of units, $g=\left(g_{x}\right)_{x \in U}$ is the vector that presents the highest amount of all energy grades for each unit, and $E: G^{U} \rightarrow \mathbb{R}$ is a usability map with $E\left(0_{U}\right)=0$ which assigns to each $\sigma=\left(\sigma_{x}\right)_{x \in U} \in G^{U}$ the usability that the units can display when each unit $x$ operates at energy grade $\sigma_{x}$. As $g \in \mathbb{R}^{U}$ is fixed throughout this article, we write $(U, E)$ rather than $(U, g, E)$.

Denote the class of all multi-choice models by $\mathcal{M C F}$. Given $(U, E) \in \mathcal{M C F}$ and $\sigma \in G^{U}$, we write $M(\sigma)=\left\{x \in U \mid \sigma_{x} \neq 0\right\}, \sigma_{D}$ as the restriction of $\sigma$ at $D$ for each $D \subseteq U$ and $\|\sigma\|=\sum_{x \in U} \sigma_{x}$.

Given $(U, E) \in \mathcal{M C F}$, let $J^{U}=\left\{\left(x, k_{x}\right) \mid x \in U, k_{x} \in G_{x}^{+}\right\}$. An allocation rule on $\mathcal{M C F}$ is a map $\phi$ that assigns to each $(U, E) \in \mathcal{M C F}$ an element

$$
\phi(U, E)=\left(\phi_{x, k_{x}}(U, E)\right)_{\left(x, k_{x}\right) \in J^{U}} \in \mathbb{R}^{J^{U}}
$$

where $\phi_{x, k_{x}}(U, E)$ is the value or the payoff of the unit $x$ when it operates in a coalition with grade $k_{x}$ in $(U, E)$. For convenience, one could define that $\phi_{x, 0}(U, E)=0$ for each $x \in U$.

A multi-choice analogue of the PEANSC in accordance with Hsieh and Liao [3] is defined as follows.

Definition 1. The cumulative individual rule (CIDR), $\Phi$, is the map on $\mathcal{M C F}$ which associates to each $(U, E) \in \mathcal{M C F}$, each unit $x \in U$, and each $k_{x} \in G_{x}^{+}$the value

$$
\bar{\Phi}_{x, k_{x}}(U, E)=\Phi_{x, k_{x}}(U, E)+\frac{1}{\|g\|} \cdot\left[E(g)-\sum_{t \in U} \sum_{k_{t}=1}^{g_{t}} \Phi_{t, k_{t}}(U, E)\right],
$$

where $\Phi_{x, k_{x}}(U, E)=E\left(k_{x}, 0_{U \backslash\{x\}}\right)$ is the cumulative single-grade effect of the unit $x$ from its grade 0 to $k_{x}$. Under the allocation rule $\bar{\Phi}$, units first take their cumulative single-grade effects under corresponding grades and further distribute the remaining resources equally among all grades.

In the following, a motivating example is provided to show how the concepts of the multi-choice model and the CIDR could be applied and to clarify our meaning.

Example 1. In a resource-allocation process for a sports organization, the formulation of relevant plans has a critical influence on the allocation management trend of the whole process. There are plenty of different members in the strategy-making meetings, such as marketing experts, physical education experts, resource management experts, sports science experts, and so on. Suppose that $U$ is the set of all members in the strategy-making meetings of a resource-allocation process. Each member $x$ could have different strategy-making choices $\sigma_{x} \in G_{x}$; each member will strategically align with other members due to different plans and adopt different strategy-making behaviors for different plans and different members. Thus, a mapping $E$ could be applied to evaluate the utilities of strategies adopted by all members against different plans (i.e., $E(\sigma), \sigma \in G^{U}$ ). Nevertheless, there is almost no single plan that can be discussed and formulated in the whole strategy-making meeting, such as resource-allocation policies, teaching implementation plans, equipment design programs, and so on. Therefore, the strategy-making meetings of the resource-allocation processes can be regarded as a multi-choice strategy-making model $(U, E)$. To evaluate the influence of each member in the strategy-making meetings, using the allocation rules proposed in this work, this article first assesses the cumulative individual-grade contribution that each member has averaged over previous meetings based on numerous and distinct behaviors, which is the cumulative single-grade effect 
$\Phi$ defined in Definition 1. The remaining shared resources should also be allocated equally by all members, which is the cumulative individual rule $\bar{\Phi}$ defined in Definition 1.

Consequently, it is important to encourage members to utilize relevant strategies for all resources, so that limited resources can result in the most proportionate productivity. By applying the characterizations of the MWII, all the resources and utilities are accumulated and evaluated under the mode that managers adopt multiple strategies. It is expected that the following axiomatic and dynamic results of the CIDR could be used to achieve beneficial outcomes for the analysis of the strategic effects of all members in the strategy-making meetings of resource-allocation processes.

In the following, a numerical example is provided. Let $(U, E) \in \mathcal{M C F}$ be a resourcedistributing situation, where $U=\{x, y, z\}$ is the set of units, $g=(1,1,2)$ is the operational grade vector that presents the highest amount of all energy grades for each unit, and $E: G^{U} \rightarrow \mathbb{R}$ is a usability mapping with $E(0,0,0)=0$ which assigns to each $\sigma=\left(\sigma_{t}\right)_{t \in U}$ the usability that the units can exhibit when each unit $t$ operates at energy grade $\sigma_{t}$. Further, assume that $E(1,1,2)=12, E(1,1,1)=2, E(1,1,0)=-3, E(1,0,1)=4, E(1,0,2)=9$, $E(0,1,1)=-5, E(0,1,2)=7, E(1,0,0)=-2, E(0,1,0)=3, E(0,0,2)=4, E(0,0,1)=-1$, and $E(0,0,0)=0$ are the usabilities that the units can exhibit under all operational grades. Based on Definition 1,

$$
\begin{aligned}
& \Phi_{x, 1}(U, E)=-2, \Phi_{y, 1}(U, E)=3, \Phi_{z, 2}(U, E)=4, \Phi_{z, 1}(U, E)=-1 \\
& \Phi_{x, 1}(U, E)=0, \Phi_{y, 1}(U, E)=5, \Phi_{z, 2}(U, E)=6, \Phi_{z, 1}(U, E)=1 .
\end{aligned}
$$

The value of each unit when it takes a specific grade in $(U, E)$ is clear. For example, the value of unit $z$ is $\overline{\Phi_{z, 2}}(U, E)=6$ when $z$ takes grade 2 in $(U, E)$.

\section{Axiomatic Processes}

This section demonstrates that the CIDR could be characterized by some useful axioms, showing the rationality of the CIDR. Therefore, some useful axiomatic properties are required. Let $\phi$ be an allocation rule on $\mathcal{M C F}$.

- $\phi$ satisfies grade completeness (GCLS) if $\sum_{x \in U} \sum_{k_{x}=1}^{g x} \phi_{x, k_{x}}(U, E)=E(g)$ for all $(U, E) \in \mathcal{M C F}$.

- $\quad \phi$ satisfies criterion for models (CFM) if $\phi(U, E)=\bar{\Phi}(U, E)$ for all $(U, E) \in \mathcal{M C F}$ with $|U| \leq 2$.

- $\quad \phi$ satisfies unmixed equality symmetry (UES) if for all $(U, E) \in \mathcal{M C F}$ and for all $\sigma \in G^{U}$ with $E\left(k_{x}, 0, \sigma_{N \backslash\{x, y\}}\right)=E\left(0, k_{y}, \sigma_{N \backslash\{x, y\}}\right)$ for some $\left(x, k_{x}\right),\left(y, k_{y}\right) \in J^{U}$, $\phi_{x, k_{x}}(U, E)=\phi_{y, k_{y}}(U, E)$.

- $\quad \phi$ satisfies grade synchronization (GSRN) if for all $(U, E),(U, D) \in \mathcal{M C F}$ with $E(\sigma)=D(\sigma)+\sum_{x \in M(\sigma)} \sum_{k_{x}=1}^{\sigma_{x}} \mu_{x, k_{x}}$ for some $\mu \in \mathbb{R}^{J^{U}}$ and for all $\sigma \in G^{U}, \phi(U, E)=$ $\phi(U, D)+\mu$.

GCLS states that all units allocate all resources entirely in a model. CFM is a selfsufficient situation if there exists only one unit in the model, but if there are two units in the model, each of them first gains what they could have caused alone, and at the end of the acting process, they share all the remaining losses and profits. UES states that the values of two energy grades should be coincident if the contributions of these two units are equal. GSRN can be asserted to be a significant weakness of additivity. The interaction between the above properties and resource-allocation processes is presented in Section 5. It is easy to show that the CIDR satisfies GCLS, CFM, UES, and GSRN according to Definition 1. In Section 5 , the interaction between the above axiomatic properties and resource-allocation processes is presented in detail. 
A multi-choice analogue of the reduction according to Hsieh and Liao [3] is considered as follows. Given $(U, E) \in \mathcal{M C F}, H \subseteq U$ and an allocation rule $\phi$, the reduced model $\left(H, E_{H}^{\phi}\right)$ is defined by

$$
E_{H}^{\phi}(\sigma)= \begin{cases}0 & \sigma=0_{H}, \\ E\left(\sigma_{x}, 0_{U \backslash\{x\}}\right) & H \geq|2|, M(\sigma)=\{x\} \text { for some } x, \\ E\left(\sigma, g_{U \backslash H}\right)-\sum_{x \in U \backslash H} \sum_{k_{x}=1}^{g_{x}} \phi_{x, k_{x}}(U, E) & \text { otherwise, }\end{cases}
$$

for all $\sigma \in G^{H}$. The consistency property may be asserted as follows. Let $\phi$ be an allocation rule on $\mathcal{M C F}$. For any pair of two units in a model, one introduces a "reduced model" among them by verifying the amounts remaining after the rest of the units are given the effects assigned from $\phi$. Then, $\phi$ is consistent if the same effects usually emerge as in the initial model when it is applied to the arbitrary reduced model. Formally, an allocation rule $\phi$ satisfies consistency (CSY) if for all $(U, E) \in \mathcal{M C F}$ with $|U| \geq 3$, for all $H \subseteq U$ with $|H|=2$, and for all $\left(x, k_{x}\right) \in J^{H}, \phi_{x, k_{x}}(U, E)=\phi_{x, k_{x}}\left(H, E_{H}^{\phi}\right)$.

Subsequently, some results can be presented after adopting the CSY property. The following result shows the consistency of the CIDR.

Lemma 1. The CIDR $\bar{\Phi}$ satisfies CSY.

Proof. Let $(U, E) \in \mathcal{M C F}$ with $|U| \geq 3$ and $H \subseteq U$ with $|H|=2$. Assume that $H=\{x, h\}$. By the definition of $\bar{\Phi}$, for all $\left(x, k_{x}\right) \in J^{H}$,

$$
\bar{\Phi}_{x, k_{x}}\left(H, E_{H}^{\bar{\Phi}}\right)=\Phi_{x, k_{x}}\left(H, E_{H}^{\bar{\Phi}}\right)+\frac{s\left(k_{x}\right)}{\left\|g_{H}\right\|} \cdot\left[E_{H}^{\bar{\Phi}}\left(g_{H}\right)-\sum_{t \in H} \sum_{k_{t}=1}^{g_{t}} \Phi_{t, k_{t}}\left(H, E_{H}^{\bar{\Phi}}\right)\right] .
$$

By definitions of $\Phi$ and $E_{H}^{\Phi}$, for all $k_{x} \in G_{x}^{+}$,

$$
\Phi_{x, k_{x}}\left(H, E_{H}^{\bar{\Phi}}\right)=E_{H}^{\bar{\Phi}}\left(k_{x}, 0\right)=E\left(k_{x}, 0_{U \backslash\{x\}}\right)=\Phi_{x, k_{x}}(U, E) .
$$

Hence, by Equations (1) and (2) and definitions of $E_{H}^{\bar{\Phi}}$ and $\bar{\Phi}$,

$$
\begin{aligned}
\bar{\Phi}_{x, k_{x}}\left(H, E_{H}^{\bar{\Phi}}\right) & =\Phi_{x, k_{x}}(U, E)+\frac{1}{\left\|g_{H}\right\|} \cdot\left[E_{H}^{\Phi}\left(g_{H}\right)-\sum_{t \in H} \sum_{k_{t}=1}^{g_{t}} \Phi_{t, k_{t}}(U, E)\right] \\
& =\Phi_{x, k_{x}}(U, E)+\frac{1}{\left\|g_{H}\right\|} \cdot\left[E(g)-\sum_{t \in U \backslash H} \sum_{k_{t}=1}^{g_{t}} \bar{\Phi}_{t, k_{t}}(U, E)-\sum_{t \in H} \sum_{k_{t}=1}^{g_{t}} \Phi_{t, k_{t}}(U, E)\right] \\
& =\Phi_{x, k_{x}}(U, E)+\frac{1}{\left\|g_{H}\right\|} \cdot\left[\sum_{t \in H} \sum_{k_{t}=1}^{g_{t}} \bar{\Phi}_{t, k_{t}}(U, E)-\sum_{t \in H} \sum_{k_{t}=1}^{g_{t}} \Phi_{t, k_{t}}(U, E)\right] \\
& =\Phi_{x, k_{x}}(U, E)+\frac{1}{\left\|g_{H}\right\|} \cdot\left[\frac{\left\|g_{H}\right\|}{\|g\|} \cdot\left[E(g)-\sum_{t \in U} \sum_{k_{t}=1}^{g_{t}} \Phi_{t, k_{t}}(U, E)\right]\right] \\
& =\Phi_{x, k_{x}}(U, E)+\frac{1}{\|g\|} \cdot\left[E(g)-\sum_{t \in U} \sum_{k_{t}=1}^{g_{t}} \Phi_{t, k_{t}}(U, E)\right] \\
& =\bar{\Phi}_{x, k_{x}}(U, E) .
\end{aligned}
$$

That is, the CIDR satisfies CSY.

The following result shows that the grade completeness property could be replaced by the properties of criteria for models and consistency.

Lemma 2. If an allocation rule $\phi$ satisfies CFM and CSY then it also satisfies GCLS. 
Proof. Let $\phi$ be an allocation rule on $\mathcal{M C F}$ satisfying CFM and CSY, and $(U, E) \in \mathcal{M C F}$. It is trivial for $|U| \leq 2$ by CFM. Assume that $|U| \geq 3$. Let $t \in U$, consider the reduced model $\left(\{t\}, E_{\{t\}}^{\phi}\right)$. By definition of $E_{\{t\}}^{\phi}, E_{\{t\}}^{\phi}\left(g_{t}\right)=E(g)-\sum_{x \in U \backslash\{t\}} \sum_{k_{x}=1}^{g_{x}} \phi_{x, k_{x}}(U, E)$. Since $\phi$ satisfies CSY, $\phi_{t, k_{t}}(U, E)=\phi_{t, k_{t}}\left(\{t\}, E_{\{t\}}^{\phi}\right)$ for all $k_{t} \in G_{t}$. In particular, $\phi_{t, g_{t}}(U, E)=$ $\phi_{t, g_{t}}\left(\{t\}, E_{\{t\}}^{\phi}\right)$. On the other hand, by CFM of $\phi, \sum_{k_{t}=1}^{g_{t}} \phi_{t, k_{t}}(U, E)=E_{\{t\}}^{\phi}\left(g_{t}\right)$. Hence, $\sum_{x \in U} \sum_{k_{x}=1}^{g_{x}} \phi_{x, k_{x}}(U, E)=E(g)$, i.e., $\phi$ satisfies GCLS.

Inspired by Hart and Mas-Colell [8] and Moulin [9], the CIDR can be characterized by the properties of criteria for models and consistency.

Theorem 1. An allocation rule $\phi$ on $\mathcal{M C F}$ satisfies CFM and CSY if and only if $\phi=\bar{\Phi}$.

Proof. By Lemma $1, \bar{\Phi}$ satisfies CSY. Clearly, $\bar{\Phi}$ satisfies CFM.

To demonstrate uniqueness, suppose that $\phi$ satisfies CFM and CSY on $\mathcal{M C F}$. By Lemma 2, $\phi$ also satisfies GCLS. Let $(U, E) \in \mathcal{M C F}$. If $|U| \leq 2$, then by CFM of $\phi$, $\phi(U, E)=\bar{\Phi}(U, E)$. For the case $|U|>2$, let $x \in U$ and $H=\{x, y\}$ for some $y \in U \backslash\{x\}$. For all $k_{x} \in G_{x}^{+}$,

$$
\begin{array}{rlrl}
\Phi_{x, k_{x}}\left(H, E_{H}^{\phi}\right) & =E_{H}^{\phi}\left(k_{x}, 0\right) & \\
& =E\left(k_{x}, 0 u \backslash\{x\}\right) & & \left(\text { By definition of } E_{H}^{\phi}\right) \\
& =E_{H}^{\bar{\Phi}}\left(k_{x}, 0\right) & & \left(\text { By definition of } E_{H}^{\Phi}\right) \\
& =\Phi_{x, k_{x}}\left(H, E_{H}^{\Phi}\right) . &
\end{array}
$$

Further,

$$
\begin{array}{rlr}
\phi_{x, k_{x}}(U, E)-\bar{\Phi}_{x, k_{x}}(U, E) & =\phi_{x, k_{x}}\left(H, E_{H}^{\phi}\right)-\bar{\Phi}_{x, k_{x}}\left(H, E_{H}^{\bar{\Phi}}\right) & (\text { By CSC of } \phi \text { and } \bar{\Phi}) \\
& =\bar{\Phi}_{x, k_{x}}\left(H, E_{H}^{\phi}\right)-\bar{\Phi}_{x, k_{x}}\left(H, E_{H}^{\Phi}\right) & (\text { By CFM of } \phi \text { and } \bar{\Phi}) \\
& =\frac{1}{\left\|g_{H}\right\|}\left[E_{H}^{\phi}\left(g_{H}\right)-E_{H}^{\bar{\Phi}}\left(g_{H}\right)\right] . & (\text { By Equation (3) and CFM of } \phi \text { and } \bar{\Phi})
\end{array}
$$

Similarly, for all $k_{y} \in G_{y}^{+}$,

$$
\phi_{y, k_{y}}(U, E)-\bar{\Phi}_{y, k_{y}}(U, E)=\frac{1}{\left\|g_{H}\right\|}\left[E_{H}^{\phi}\left(g_{H}\right)-E_{H}^{\bar{\Phi}}\left(g_{H}\right)\right] .
$$

By Equations (4) and (5), $\phi_{x, k_{x}}(U, E)-\bar{\Phi}_{x, k_{x}}(U, E)=\phi_{y, k_{y}}(U, E)-\bar{\Phi}_{y, k_{y}}(U, E)$. This implies that $\phi_{x, k_{x}}(U, E)-\bar{\Phi}_{x, k_{x}}(U, E)=c$ for all $\left(x, k_{x}\right)$. It remains to demonstrate that $c=0$. By GCLS of $\phi$ and $\Phi$,

$$
\begin{aligned}
0 & =E(g)-E(g) \\
& =\sum_{x \in U} \sum_{k_{x}=1}^{g_{x}}\left[\phi_{x, k_{x}}(U, E)-\bar{\Phi}_{x, k_{x}}(U, E)\right] \\
& =c \cdot\|g\| \cdot\left[\phi_{x, k_{x}}(U, E)-\bar{\Phi}_{x, k_{x}}(U, E)\right] .
\end{aligned}
$$

That is, $c=0$.

The following result shows that the criteria for model properties could be replaced by the properties of grade completeness, unmixed equality symmetry, and grade synchronization.

Lemma 3. If an allocation rule $\phi$ on $\mathcal{M C F}$ satisfies GCLS, UES, and GSRN, then $\phi$ satisfies CFM. 
Proof. Assume that an allocation rule $\phi$ satisfies GCLS, UES, and GSRN. Let $(U, E) \in$ $\mathcal{M C F}$. The proof of $|U|=1$ is finished by GCLS of $\phi$. Let $U=\{x, h\}$ for some $x \neq h$. A model $(U, D)$ is defined so that for all $\sigma \in G^{U}$,

$$
D(\sigma)=E(\sigma)-\sum_{t \in U} \sum_{k_{t}=1}^{\sigma_{t}} E\left(k_{t}, 0_{U \backslash\{t\}}\right)
$$

Clearly, for all $k_{x} \in G_{x}^{+}$and for all $k_{y} \in G_{y}^{+}, D\left(k_{x}, 0,0_{U \backslash\{x, y\}}\right)=0=D\left(0, k_{y}, 0_{U \backslash\{x, y\}}\right)$, i.e., $D\left(k_{x}, 0, \sigma_{N \backslash\{x, y\}}\right)=D\left(0, k_{y}, \sigma_{N \backslash\{x, y\}}\right)$ for all $\sigma \in G^{U}$. By GCLS of $\phi$,

$$
D(g)=\sum_{k_{x}=1}^{g_{x}} \phi_{x, k_{x}}(U, D)+\sum_{k_{h}=1}^{g_{h}} \phi_{h, k_{h}}(U, D)=\|g\| \cdot \phi_{x, k_{x}}(U, D)
$$

for all $k_{x} \in G_{x}^{+}$. By GSRN of $\phi$, Equation (6) and definition of $D$,

$$
\begin{aligned}
\phi_{x, k_{x}}(U, E) & =E\left(k_{x}, 0\right)+\frac{1}{\|g\|} \cdot\left[E(g)-\sum_{t \in U} \sum_{k_{t}=1}^{g_{t}} E\left(k_{t}, 0\right)\right] \\
& =\Phi_{x, k_{x}}(U, E)+\frac{1}{\|g\|} \cdot\left[E(g)-\sum_{t \in U} \sum_{k_{t}=1}^{g_{t}} \Phi_{t, k_{t}}(U, E)\right]
\end{aligned}
$$

Hence, $\phi$ satisfies CFM.

Inspired by Maschler and Owen [10], one may characterize the CIDR by means of the properties of grade completeness, unmixed equality symmetry, grade synchronization, and consistency.

Theorem 2. An allocation rule $\phi$ on $\mathcal{M C F}$ satisfies GCLS, UES, GSRN, and CSY if and only if $\phi=\bar{\Phi}$.

Proof. By Definition 1, $\bar{\Phi}$ satisfies GCLS, UES, and GSRN. The rest of the proof could be completed by Theorem 1 and Lemmas 1 and 3 .

\section{Dynamic Process}

In this section, a symmetric revising function and relevant dynamic process for the CIDR are introduced. The fundamental concept of this symmetric revising function is briefly stated as follows: first, the participating unit in an allocation process solicits and picks the effective difference between the unit and all other participating units regarding whether each unit has joined or not and then revises the picked aggregate symmetrically by a modulus, finally applying this to adjust the previous value. Hence, the relevance between "the extent of a modulus" and "whether such a process can approach the CIDR" is inextricable. That is, irrespective of the extent of the modulus, the arbitrary allocation rule could be revised continuously by means of the above process, and eventually it would approach the CIDR. Subsequently, the following outcomes examine the relevance of this.

In the following, the notion of a surfeit is adopted to offer a dynamic approach that leads the units to the CIDR, starting from an arbitrary grade completeness vector. Some definitions should be provided. Let $(U, E) \in \mathcal{M C F}$. The collection of level complement outcomes on $(U, E)$ is denoted by $L G(U, E)=\left\{\alpha \in \mathbb{R}^{J^{U}} \mid \sum_{x \in U} \sum_{k_{x}=1}^{g_{i}} \alpha_{x, k_{x}}=E(g)\right\}$. Let $(U, E) \in \mathcal{M C F}$ and $\alpha \in L G(U, E)$. The surfeit of a multi-choice coalition $\lambda \in G^{U}$ at $\alpha$ is the real number

$$
E S(\lambda, E, \alpha)=\sum_{x \in U}\left[E(\lambda)-\alpha_{x, \lambda_{x}}\right]
$$


The value $E S(\lambda, E, \alpha)$ can be regarded as the holler of reactive level vector $\lambda$ when all factors obtain their effects from $\alpha$ in $(U, E)$.

Let $(U, E) \in \mathcal{M C F}, \alpha \in L G(U, E)$, and $t>0$; one can define the symmetric revising function $h: L G(U, E) \rightarrow \mathbb{R}^{J^{U}}$ as follows. $h=\left(h_{x, k_{x}}\right)_{\left(x, k_{x}\right) \in J^{u}}$ and for all $\left(x, k_{x}\right) \in J^{U}$,

$h_{x, k_{x}}(\tau(U, E))=\alpha_{x, k_{x}}+t . \sum_{y \in U \backslash\{x\}} \sum_{k_{y}=1}^{g_{y}}\left(E S\left(\left(k_{x}, 0_{U \backslash\{y\}}\right), E, \alpha\right)-E S\left(\left(k_{y}, 0_{U \backslash\{x\}}\right), E, \alpha\right)\right)$,

where $t$ is a fixed positive number, which reflects the assumption that factor $i$ does not ask for full modification (when $t=1$ ) but only (usually) a fraction of it. The number $t$ represents the degree to which the surfeit is corrected. When factors participate in a circumstance, some hollers or variations may occur in different situations. The modification functions are based on the idea that each factor shortens the holler relating to its own and others' non-participation and uses these regulations to correct the initial value.

The following lemma shows that the symmetric revising function is well-defined. This lemma also plays an essential role in obtaining the necessary convergence outcome.

Lemma 4. $h(x) \in L G(U, E)$ for all $(U, E) \in \mathcal{M C F}$ and $\alpha \in L G(U, E)$.

Proof. Let $(U, E) \in \mathcal{M C F}, \alpha \in L G(U, E)$ and $\left(x, k_{x}\right) \in J^{U}$. So,

$$
\begin{aligned}
& \sum_{y \in U \backslash\{x\}} \sum_{k_{y}=1}^{g_{y}}\left(E S\left(\left(k_{x}, 0_{U \backslash\{x\}}\right), E, \alpha\right)-E S\left(\left(k_{y}, 0_{U \backslash\{y\}}\right), E, \alpha\right)\right) \\
= & \sum_{y \in U \backslash\{x\}} \sum_{k_{y}=1}^{g_{y}}\left(E\left(k_{x}, 0_{U \backslash\{x\}}\right)-\alpha_{x, k_{x}}-E\left(k_{y}, 0_{U \backslash\{y\}}\right)+\alpha_{y, k_{y}}\right) \\
= & \sum_{y \in U \backslash\{x\}} \sum_{k_{y}=1}^{g_{y}}\left(\Phi_{x, k_{x}}(U, E)-\alpha_{x, k_{x}}-\Phi_{y, k_{y}}(U, E)+\alpha_{y, k_{y}}\right) \\
= & \sum_{y \in U \backslash\{x\}} \sum_{k_{y}=1}^{g_{y}}\left(\Phi_{x, k_{x}}(U, E)-\alpha_{x, k_{x}}-\bar{\Phi}_{y, k_{y}}(U, E)+\alpha_{y, k_{y}}\right) \\
= & \|g\| \cdot\left(\bar{\Phi}_{x, k_{x}}(U, E)-\alpha_{x, k_{x}}\right)+\sum_{y \in U} \sum_{k_{y}=1}^{g_{y}}\left(\alpha_{y, k_{y}}-\bar{\Phi}_{y, k_{y}}(U, E)\right) \\
= & \|g\| \cdot\left(\bar{\Phi}_{x, k_{x}}(U, E)-\alpha_{x, k_{x}}\right)+(E(g)-E(g)) \\
= & \|g\| \cdot\left(\bar{\Phi}_{x, k_{x}}(U, E)-\alpha_{x, k_{x}}\right) .
\end{aligned}
$$

Hence, by Equations (7) and (8),

$$
\begin{aligned}
& \sum_{x \in U} \sum_{k_{x}=1}^{g_{x}} h_{x, k_{x}}(x) \\
= & \sum_{x \in U} \sum_{k_{x}=1}^{g_{x}}\left[\alpha_{x, k_{x}}+t \cdot \sum_{y \in U \backslash\{x\}} \sum_{k_{y}=1}^{g_{y}}\left(E S\left(\left(k_{x}, 0_{U \backslash\{y\}}\right), E, \alpha\right)-E S\left(\left(k_{y}, 0_{U \backslash\{x\}}\right), E, \alpha\right)\right)\right] \\
= & \sum_{x \in U} \sum_{k_{x}=1}^{g_{x}} \alpha_{x, k_{x}}+t \cdot \sum_{x \in U} \sum_{k_{x}=1}^{g_{x}}\left[\sum_{y \in U \backslash\{x\}} \sum_{k_{y}=1}^{g_{y}}\left(E S\left(\left(k_{x}, 0_{U \backslash\{y\}}\right), E, \alpha\right)-E S\left(\left(k_{y}, 0_{U \backslash\{x\}}\right), E, \alpha\right)\right)\right] \\
= & E(g)+t \cdot\|g\| \sum_{x \in U} \sum_{k_{x}=1}^{g_{x}}\left(\bar{\Phi}_{x, k_{x}}(U, E)-\alpha_{x, k_{x}}\right) \\
= & E(g)+t \cdot\|g\|(E(g)-E(g)) \\
= & E(g) .
\end{aligned}
$$

Thus, $h(x) \in L G(U, E)$.

Let $(U, E) \in \mathcal{M C F}$ and $\alpha \in L G(U, E)$. One could define the sequences $\left\{\alpha^{q}\right\}_{q=1}^{\infty}$ so that $\alpha^{0}(U, E)=\alpha, \cdots, \alpha^{q}=h\left(\alpha^{q-1}\right)$ for all $q \in \mathbb{N}$. 
Theorem 3. If $0<t<\frac{2}{\|g\|}$, then $\left\{\alpha_{x, k_{x}}^{q}\right\}_{q=1}^{\infty}$ converges geometrically to $\bar{\Phi}_{x, k_{x}}(U, E)$ for all $(U, E) \in \mathcal{M C F}$, for all $\alpha \in L G(U, E)$ and for all $\left(x, k_{x}\right) \in J^{U}$.

Proof. Let $(U, E) \in \mathcal{M C F}$ and $\alpha \in L G(U, E)$. By Lemma 1 and the definition of $h$, for all $\left(x, k_{x}\right) \in J^{U}$,

$$
\begin{aligned}
h_{x, k_{x}}(\tau(U, E))-\alpha_{x, k_{x}} & =t \cdot \sum_{y \in U \backslash\{x\}} \sum_{k_{y}=1}^{g_{y}}\left(E S\left(\left(k_{x}, 0_{U \backslash\{y\}}\right), E, \alpha\right)-E S\left(\left(k_{y}, 0_{U \backslash\{x\}}\right), E, \alpha\right)\right) \\
& =t \cdot\|g\| \cdot\left(\Phi_{x, k_{x}}(U, E)-\alpha_{x, k_{x}}\right) .
\end{aligned}
$$

Hence,

$$
\begin{aligned}
\bar{\Phi}_{x, k_{x}}(U, E)-h_{x, k_{x}}(x) & =\bar{\Phi}_{x, k_{x}}(U, E)-\alpha_{x, k_{x}}+\alpha_{x, k_{x}}-h_{x, k_{x}}(\tau(U, E)) \\
& =\bar{\Phi}_{x, k_{x}}(U, E)-\alpha_{x, k_{x}}-t \cdot\|g\| \cdot\left(\bar{\Phi}_{x, k_{x}}(U, E)-\alpha_{x, k_{x}}\right) \\
& =(1-t \cdot\|g\|) \cdot\left[\bar{\Phi}_{x, k_{x}}(U, E)-\alpha_{x, k_{x}}\right] .
\end{aligned}
$$

So, for all $q \in \mathbb{N}, \bar{\Phi}_{x, k_{x}}(U, E)-\alpha_{x, k_{x}}^{q}=(1-t \cdot\|g\|)^{q} \cdot\left[\bar{\Phi}_{x, k_{x}}(U, E)-\alpha_{x, k_{x}}\right]$. If $0<t<\frac{2}{\|g\|}$, then $-1<(1-t \cdot\|g\|)<1$ and $\left\{\alpha_{x, k_{x}}^{q}\right\}_{q=1}^{\infty}$ converges geometrically to $\bar{\Phi}_{x, k_{x}}(U, E)$.

\section{Discussion of Resource Allocating Processes and Concluding Remarks}

Based on the current cross-disciplinary considerations among different domains, this article uses axiomatic and dynamic results to examine the accuracy and the plausibility of resource-allocation rule by asking the following questions: "how is this rule defined?", "why does one focus on this rule?", "is this rule correct'?' and "how suitable is this rule?".

In Sections 2 and 3, we show that the major merit of the CIDR is that the CIDR of a multi-choice model exists exactly and results in an exact value for a specific unit that participates with a specific energy grade; this is in contrast with the general proposition of multi-choice models, which result in a type of while value for a specific unit by picking the marginal contributions of the unit among its whole energy grades. One would like to expect that the CIDR could result in an exact "apposite outcome" for resource-allocation processes. The interaction among axiomatic properties and resource-allocation processes can be further investigated so that the usage of the CIDR could be presented with a clearer definition.

1. Grade completeness: Apposite resource-allocation processes should make full use of all resources. Hence, an apposite resource-allocation process should fulfill the grade completeness property.

2. Criterion for models: Units own their specific activity characteristics. Interactions among units are usually derived from two-unit interactions followed by a group effect. Thus, an apposite resource-allocation process should match the criteria for model properties.

3. Unmixed equality symmetry: If any two units are equally unmixed in the situation after the action of unit grouping, the influence of these two units on the whole should also be equal. Accordingly, an apposite resource-allocation process should fulfill the unmixed equality symmetry property.

4. Grade synchronization: Apposite resource-allocation processes, in which each unit is applied with the appropriate energy grade to reach a target rather than a amount (large or small) depending its energy grade, should fulfill the most apposite efficacy in accordance with the proportionality principle. Hence, an apposite resource-allocation process should satisfy the grade synchronization property. 
5. Consistency: Apposite resource-allocation processes should be examined via an iterative continuous procedure and should produce consistent benefits. An apposite resource-allocation process should therefore satisfy the consistency property.

As mentioned in Section 2, it is possible to show that the framework of resourceallocation processes could be formulated as a multi-choice model. By applying Theorems 1 and 2, it is shown that the CIDR is the only allocation rule that simultaneously satisfies grade completeness, criteria for models, unmixed equality symmetry, grade synchronization, and consistency. By statements $1-5$, it is also clear that the properties of grade completeness, criteria for models, unmixed equality symmetry, grade synchronization, and consistency are indispensable requirements in resource-allocation processes. Hence, the CIDR might be applied as apposite approach to resource-allocation processes.

In summary, the aim of this article is to present different discussions of resourceallocation processes.

- A generalization of the PEANSC, the cumulative individual rule, is defined for multichoice side-payment models by simultaneously considering the units and their energy grades.

- Some useful axioms are adopted to characterize the cumulative individual rules in order to dissect their mathematical accuracy and applied rationality.

- A symmetric revising function is defined to establish an interactive process that can be dynamically modified to approach the cumulative individual rule at any time under resource-allocation processes.

- In conjunction with the axiomatic and dynamic processes related to resource-allocation situations, the applicability and plausibility of the cumulative individual rules are further analyzed and demonstrated by applying some examples and interpretations.

One might compare our results with pre-existing results. Several primary dissimilarities are as follows:

- In the framework of traditional side-payment models, allocation rules have only focused on non-participation or participation among all units. As mentioned above, however, it is rational that every unit might have different energy grades. Therefore, in contrast to the PEANSC defined by Hsieh and Liao [3] on traditional side-payment models, the cumulative individual rule is defined to resolve a resource-allocation mechanism under multi-choice consideration.

- The cumulative individual rule does not appear in existing research. The axiomatic notion is a multi-choice analogue of related results due to Hart and Mas-Colell [8], Maschler and Owen [10], and Moulin [9]. Inspired by Maschler and Owen [10], a dynamic result is also introduced. The primary discrimination is that the revising function of this article arises from a "surfeit", and Maschler and Owen's [10] correction mapping originates from "reduction".

- By considering real-world situations, the cumulative individual rule is defined to calculate the associated effect of a given unit if it operates with a given energy grade. Under multi-choice models, existing allocation rules have been defined to calculate a kind of entire effect for a given unit by assembling the marginal contributions of this unit among its energy grades. As mentioned in the Introduction, Branzei [4], Liao [5] and Nouweland et al. [6] defined related allocation rules to generate several kinds of global effects for a specific unit by collecting related contributions of the unit among all of its energy grades.

1. Inspired by the core of traditional side-payment models, Branzei [4] proposed some core concepts by adopting the convexity and the stability among units and their energy grades. In contrast to Branzei [4], this article considers the notion of the PEANSC by both applying the units and their energy grades. The other primary discrimination is the fact that this article offers the properties of unmixed equality symmetry and grade synchronization to analyze the cumulative individual rule 
proposed in this article. Related properties of unmixed equality symmetry and grade synchronization are not presented in Branzei [4].

2. Inspired by the EANSC for traditional side-payment models, Liao [5] proposed the maximal EANSC by adopting the maximal pure effects of units among their energy grades. In contrast to Liao [5], this article considers the notion of the PEANSC by both applying the units and their energy grades. The other primary discrimination is the fact that this article offers the properties of unmixed equality symmetry and grade synchronization to analyze the cumulative individual rule proposed in this article. Related properties of unmixed equality symmetry and grade synchronization are not presented in Liao [5].

3. Inspired by the Shapley value for traditional side-payment models, Nouweland et al. [6] defined the multi-choice Shapley value by adopting the replicated behavior due to the units and their energy grades. Differing from Nouweland et al. [6], this article considers the notion of the PEANSC by both applying the units and their energy grades. The other primary discrimination is the fact that this article offers the properties of unmixed equality symmetry and grade synchronization to analyze the cumulative individual rule proposed in this article. Related properties of unmixed equality symmetry and grade synchronization are not presented in Nouweland et al. [6].

- $\quad$ By both considering the units and their energy grades, Hwang and Li [7] presented an extended core for multi-choice non-side-payment models. Similar to Hwang and Li [7], the cumulative individual rule of this article is defined by both considering the units and their energy grades under multi-choice side-payment models. One should also compare our work with the outcomes of Hwang and Li [7]. There are several primary dissimilarities:

1. The allocation rule developed by Hwang and Liao [7] is based on multi-choice non-side-payment behavior. The cumulative individual rule of this article is based on multi-choice side-payment behavior.

2. The allocation rule developed by Hwang and Liao [7] is an analogue of the core. The cumulative individual rule of this article is an analogue of the PEANSC.

- The axiomatic and dynamic results of this article have been used to evaluate resourceallocation processes. This application does not appear in existing research.

One further motivation can be taken from the outcomes of this article: it should be determined whether other allocation rules and their axiomatic and dynamic results could be applied to derive the most efficient and apposite mechanisms for resource-allocation processes. This problem is left to the readers of this article.

Author Contributions: Conceptualization, Y.-H.L. and C.-H.L.; methodology, Y.-H.L. and E.-C.C.; validation, Y.-H.L. and J.-W.C.; formal analysis, Y.-H.L.; investigation, Y.-H.L.; writing-original draft preparation, Y.-H.L.; writing-review and editing, Y.-H.L., C.-H.L., J.-W.C. and E.-C.C. All authors have read and agreed to the published version of the manuscript.

Funding: This research received no external funding.

Institutional Review Board Statement: Not applicable.

Informed Consent Statement: Not applicable.

Data Availability Statement: Data available on request due to privacy restrictions. The data presented in this study are available on request from the corresponding author.

Conflicts of Interest: The authors declare no conflict of interest. 


\section{References}

1. Shapley, L.S. A value for n-person game. In Distinctions to the Theory of Games II; Kuhn, H.W., Tucker, A.W., Eds.; Princeton Press: Princeton, NJ, USA, 1953; pp. 307-317.

2. Ransmeier, J.S. The Tennessee Valley Authority; Vanderbilt University Press: Nashville, TN, USA, 1942.

3. Hsieh, Y.L.; Liao, Y.H. The Pseudo EANSC: Axiomatization and Dynamic Process. Master's Thesis, Department of Applied Mathematics, National Pingtung University, Pingtung Count, Taiwan, 2016.

4. Branzei, R.; Llorca, N.; Sanchez-Soriano, J.; Tijs, S. Multi-choice clan games and their core. TOP 2009, 17, 123-138. [CrossRef]

5. Liao, Y.H. The maximal equal allocation of nonseparable costs on multi-choice games. Econ. Bull. 2008, 3, 1-8.

6. Van den Nouweland, A.; Potters, J.; Tijs, S.; Zarzuelo, J. Core and related solution concepts for multi-choice games. ZOR-Math. Methods Oper. Res. 1995, 41, 289-311. [CrossRef]

7. Hwang, Y.A.; Li, W.H The core of multi-choice NTU games. Math. Methods Oper. Res. 2005, 61, 33-40. [CrossRef]

8. Hart, S.; Mas-Colell, A. Potential, value and consistency. Econometrica 1989, 57, 589-614. [CrossRef]

9. Moulin, H. The separability axiom and equal-sharing methods. J. Econ. Theory 1985, 36, 120-148. [CrossRef]

10. Maschler, M.; Owen, G. The consistent Shapley value for hyperplane games. Int. J. Game Theory 1989, 18, 389-407. [CrossRef] 